\title{
Two-orthogonal-view method for quantification of rad dose to neck lesions in thyroid cancer therapy patients
}

\author{
Kenneth F. Koral, Ronald S. Adler, James E. Carey, Robert C. Kline, and William \\ $H$. Beierwaltes \\ Department of Internal Medicine, Division of Nuclear Medicine, University of Michigan Medical Center, Ann \\ Arbor, Michigan 48109
}

(Received 24 September 1981; accepted for publication 11 March 1982)

\begin{abstract}
A new technique is described for the determination of the dose in rad to metastatic lesions in the neck following administration of ${ }^{131} I$ for the treatment of thyroid carcinoma. The technique utilizes a high-energy pinhole collimator mounted on an Anger camera which can be rotated to record lateral and anterior views from known positions relative to the patient. A new algorithm is derived for the determination of lesion location in three dimensions, utilizing the distance of closest approach between back-projected rays from the two views. In addition, a new derivation is presented for the estimation of ellipsoid volume from two pinhole projections. The method makes use of a computer edge detection program to delineate the projected lesion boundaries. The output is either a mean estimate from one view or a more definitive upper bound derived from both views. The uptake in counts is determined from daily anterior images which are corrected for pinhole efficiency falloff with distance. This uptake is converted into microcuries by use of an inair calibration curve. An attenuation correction is applied using the calculated lesion depth and an assumed attenuation coefficient. The dose rate is calculated using standard assumptions and is integrated graphically over the first few days when the rate peaks and by utilizing a singledecaying-exponential fit to the data over the remaining days. Illustrative patient data are presented in which doses are calculated for three neck lesions after an administration of $175 \mathrm{mCi}$ of ${ }^{131} \mathrm{I}$. These doses include a lower bound of $2400 \mathrm{rad}$ and estimates of 5300 and $9500 \mathrm{rad}$.
\end{abstract}

\section{INTRODUCTION}

The quantification of the absorbed dose in rads delivered by therapeutic activities of ingested ${ }^{131} I$ to metastatic lesions in thyroid cancer patients has been pursued for at least $11 \mathrm{yr}$. This quantification ideally requires determining the spatial distribution of the radionuclide as a function of time so that a complete dosimetric calculation can be carried out. The usual simplification, which we also will make, is to assume a simple geometric object for the lesion and a uniform distribution of isotope within the lesion. Then, the task is to measure the uptake for the lesion as a whole and to estimate its volume. To do this task, Scott et al. ${ }^{1}$ employed a scanner to record anterior and lateral views. They calibrated their uptake via a source immersed in a water bath and represented the activity as an ellipse in both views with "of course, one axis common to both the anterior and the lateral visualization." It appears that this means that they assumed the lesion was an ellipsoid whose axes were parallel and perpendicular to their orthogonal views. Thomas et al. ${ }^{2}$ used an opposed view method for computing an uptake and estimated size from anterior and lateral images taken with a high-resolution, high-energy, parallel-hole collimator mounted on an Anger camera. They measured the transmission through the patient as part of the technique. Results of their technique are presented for 12 patients. ${ }^{3}$

In this paper, we restrict ourselves to the consideration of neck lesions. We then take anterior and lateral views as previous investigators did, but we introduce the use of a pinhole collimator mounted on an Anger camera for the purpose of magnifying the image of the lesion onto the camera in at least the anterior view. In this way, we are able to utilize a commerical second-derivative edge-detection algorithm because we now satisfy the requirement for sizing accuracy of this algorithm: that the image be greater than, or equal to, the system resolution: pinhole "plus" camera. "The use of the computer for delineating the lesion extent should remove operator bias. The sizes are scaled down to true sizes by means of a measurement of the image magnifications.

The measurement of magnification is provided by a procedure which locates centers of the lesions in three dimensions. The procedure is based on taking two views of an immobile patient knowing the relative position of the camera between views. One realization of this procedure which we have used depends on a rotating Anger camera such as that used in transverse section imaging. ${ }^{5}$ Another possible method to get the two views would involve rotation of a seated patient by a suitable device and the use of a conventional camera. The procedure for lesion location can also handle the situation sometimes encountered with multiple lesions wherein the lesion projections overlap in one of the views.

Pinhole images are used in this technique for all successive-day uptake measurements. Since camera efficiency for a pinhole collimator is very dependent on the distance of the lesion in front of the pinhole, especially for small distances, accurate patient repositioning is critical. We have found that keeping the distance from skin surface to collimator constant, as given by a physical measurement as well as trying to reproduce previously recorded images, is the best technique.

The last innovation of this paper is a derivation which 
places an upper bound on the ellipsoid volume without assuming anything about its orientation. This upper bound leads to a lower bound on the dose. The particular lesion must be isolated in both views to apply the results of this derivation.

The absorbed dose calculations followed the MIRD formalism. ${ }^{6}$ The absorbed fraction for nonpenetrating beta radiation was assumed to equal 1 . The absorbed fraction for penetrating gamma radiation will vary depending on the energy of the gamma ray, the mass of the lesion, and the lesion geometry. For 364-keV gamma radiation, $\phi$ varies from approximately $0.01-0.05$ for $2-60-\mathrm{g}$ small unit-density spheres or ellipsoids surrounded by scattering medium. ${ }^{7}$ For the purpose of these calculations, it was assumed to equal 0.03. $\mathrm{Cu}$ mulated activity was obtained by integration under the time activity curve, with the later data points fit with a single exponential curve so that time integration to infinity can be carried out.

\section{METHOD}

\section{A. Location of lesions in three dimensions}

In the following, we will describe the geometry for a rotating camera system. The extension to the rotating patient system will not be explicitly covered but could be derived from modifying the present discussion.

First of all, we assume a well-behaved rotating camera such that the camera diameter which is parallel to the $y$ or "vertical" image axis is also parallel to the axis of rotation. Moreover, we assume that the image offsets are adjusted so that the middle of the camera coincides with the middle of the image. We also assume that the center of the pinhole in the pinhole collimator lies directly over the center of the camera. Finally, we assume that the camera rotates through exactly $90^{\circ}$ between the anterior and lateral view. All these conditions are likely to exist for a rotating camera being used for emission computed tomography.

Given these assumptions, perpendiculars extending from the camera center through the center of the pinhole in the anterior position and in the lateral position will intersect each other and the axis of rotation. The intersection point is the origin of our coordinate system, and the two perpendiculars plus the axis of rotation make up the coordinate axes (see Fig. 1).

We also assume that the camera can travel toward and away from its axis of rotation without any other displacement and that a scale is available to measure the relative motion. For the pinhole collimator used, it is necessary to know the distance from the pinhole to the camera face $a$ and to measure the distance $b_{a}$ anteriorly or $b_{l}$ laterally, from the pinhole to the origin of coordinates when the camera is positioned at a known place as given by the scale reading. The latter distances are determined by taking anterior and lateral views of a marker source placed at an arbitrary position in front of the collimator. Previously, the average of the $x$ and $y$ camera gain in $\mathrm{mm} /$ matrix element has been determined by taking an image of an orthogonal hole phantom with known distance between the holes using a $99 \mathrm{~m}$ Tc field flood.

Projections of the marker into the anterior and lateral

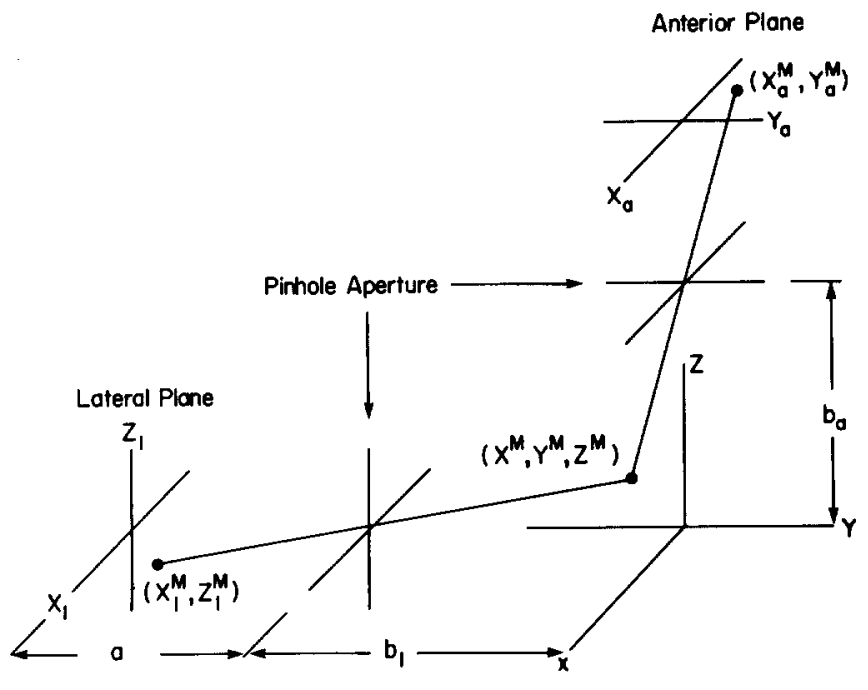

FIG. 1. Illustration of the coordinate system and marker projections. The $x$ axis represents the axis of rotation of the gamma camera. The subscripts $l$ and $a$ refer to lateral and anterior detector planes, respectively. $a$ is the detector-aperture distance. $b_{l}$ and $b_{a}$ are distances from the pinhole to the origin of coordinates. The marker whose coordinates are $\left(X^{M}, Y^{M}, Z^{M}\right)$ is assumed to be a point source with projected coordinates $\left(X_{l}^{M}, Z_{l}^{M}\right)$ and $\left(X_{a}^{M}, Y_{a}^{M}\right)$. The rays passing from the marker to their respective pinhole projections are indicated.

views are presented in Fig. 1. The measurements discussed below result in an overspecified set of equations for $b_{a}$ and $b_{l}$. Let the space coordinates of the marker by $\left(X^{M}, Y^{M}, Z^{M}\right)$ and those of the image $\left(X_{a}^{M}, Y_{a}^{M}\right)$, anteriorly, and $\left(X_{l}^{M}, Z_{l}^{M}\right)$, laterally. Simple geometrical considerations indicate that the following relations hold:

$$
\begin{aligned}
& X_{a}^{M}=\frac{a}{Z^{M}-b_{a}} X^{M}, \\
& Y_{a}^{M}=\frac{a}{Z^{M}-b_{a}} Y^{M},
\end{aligned}
$$

in the anterior projection, and

$$
\begin{aligned}
& X_{l}^{M}=-\frac{a}{Y^{M}+b_{l}} X^{M}, \\
& Z_{l}^{M}=-\frac{a}{Y^{M}+b_{l}} Z^{M},
\end{aligned}
$$

in the lateral projection.

The image coordinates of the marker are readily measured, and the pinhole-to-detector distance $a$ is assumed to be known. The distance $b_{l}+Y^{M}$ and $b_{a}-Z^{M}$ are measured relative to a fixed marker. Furthermore, the offset $b_{l}-b_{a}$ is assumed to be known or zero.

From these equations, $b_{a}$ and $b_{l}$ are readily determined. For example, calculation of $Z^{M}$ from Eq. (2b), along with the measured value of $Z^{M}-b_{a}$, results in $b_{a}$. The value of $b_{l}$ is automatically determined since the offset is known. However, separate determinations of these distances allows some compensation for measurement error.

After the above distance calibration, anterior and lateral images of the patient are taken. $\mathrm{He} / \mathrm{she}$ is placed on a low absorption patient cot, which is angled at about $30^{\circ}$ from the axis of rotation of the tomographic camera. This alignment produces an anterior view which appears rotated $30^{\circ}$ from a 
standard anterior image, but it has the advantage of allowing the pinhole collimator to get closer to the neck in the lateral view, because the pinhole end of the collimator fits between the shoulder and head of the patient.

We now derive the lesion locations in three dimensions, neglecting the finite size of the pinhole. The values of $a, b_{l}$, and $b_{a}$ are known from the above considerations and the approximate coordinates of the centers of the projected lesions are determined from their respective images. The situation is shown in Fig. 2.

The projected lesion centers are designated by the vectors $\left[\mathbf{r}_{l} i\right]=\left[x_{i}^{i}, z_{l}^{i}\right], \quad i=1,2, \ldots, N_{l}, \quad$ and $\left[\mathbf{r}_{a}^{j}\right]=\left[x_{a}^{j}, y_{a}^{j}\right]$, $j=1,2, \ldots, N_{a}$, where the subscripts $l$ and $a$ refer to lateral and anterior planes, respectively. Similarly, $N_{l}$ and $N_{a}$ are the number of projected lesions observed in each view.

Consider the backprojected rays of the vectors $\mathbf{r}_{l}^{i}, \mathbf{r}_{a}^{j}$ through their respective pinholes. These rays are expressed mathematically as

$$
\begin{aligned}
& \mathbf{R}_{l}^{i}(t)=\left[-x_{l}^{i}\left(\frac{t+b_{l}}{a}\right), t,-z_{l}^{i}\left(\frac{t+b_{l}}{a}\right)\right], \\
& \mathbf{R}_{a}^{j}(w)=\left[x_{a}^{j}\left(\frac{w-b_{a}}{a}\right), y_{a}^{j}\left(\frac{w-b_{a}}{a}\right), w\right] .
\end{aligned}
$$

The parameters $w$ and $t$ take on all values between $\pm \infty$.

If the vectors $\left(\mathbf{r}_{i}^{i}, \mathbf{r}_{a}^{j}\right)$ correspond to a particular lesion, then there exists a pair of numbers $\left(t^{*}, w^{*}\right)$ for which the two rays intersect. $\mathbf{R}_{I}^{i}\left(t^{*}\right)=\mathbf{R}_{a}^{j}\left(w^{*}\right)$. The representative lesion is then located. Two such rays generally do not intersect for several reasons: uncertainty in the center coordinates of the projected lesion, overlapping of projections, or the projections are not derived from the same lesion. One can, at most, find a set $\left(t_{i j}, w_{i j}\right)$ for which the distance between $\mathbf{R}_{i}^{i}(t)$ and $\mathbf{R}_{a}^{j}(w)$ :

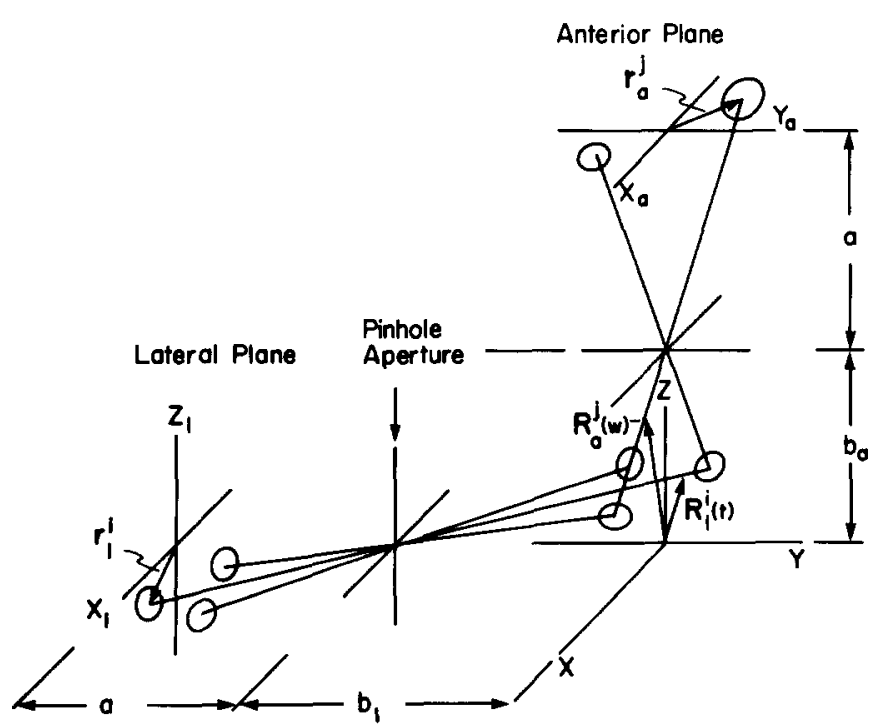

FIG. 2. Three representative lesions and their corresponding projections are depicted. $\mathbf{r}_{l}^{i}$ and $\mathbf{r}_{a}^{j}$ are vectors delineating the centers of the $i$ th projection on the lateral plane and the $j$ th projection on the anterior plane. The backprojection rays and their corresponding ray vectors $\mathbf{R}_{l}^{i}(t)$ and $\mathbf{R}_{a}^{j}(w)$ are indicated. In this idealization, the backprojected rays pass through the lesion centers. Note that the jth projection on the anterior plane is derived from the two lesion projections.

$$
S_{i j}(t, w)=\left|\mathbf{R}_{l}^{i}(t)-\mathbf{R}_{a}^{j}(w)\right|,
$$

is a minimum. The distance of closest approach $S_{i j}$ is then defined as

$$
S_{i j} \equiv S_{i j}\left(t_{i j}, w_{i j}\right) \text {. }
$$

The collections of numbers [ $\left.S_{i j}\right]$ forms a set with $N_{l} \times N_{a}$ elements corresponding to the same number of possible ray pairs. If the centers of projected lesions are located accurately, the $N$ smallest values of the $\left[S_{i j}\right]$ correspond to displacements lying entirely within individual lesions or slightly across a lesion boundary. The latter occurs only in the case of overlapping projections. Suppose the $S_{k l}$ is one of these $N$ smallest distances, and furthermore, that there is either no overlapping or overlapping in both views. The location of the lesion $\mathbf{R}$ as represented by the distance of closest approach between the rays $\mathbf{R}_{l}^{k}(t)$ and $\mathbf{R}_{a}^{l}(w)$, is taken to be

$$
\mathbf{R}=\frac{1}{2}\left[\mathbf{R}_{l}^{k}\left(t_{k l}\right)+\mathbf{R}_{a}^{l}\left(w_{k l}\right)\right] \text {. }
$$

If overlapping is observed in only one view, say anteriorly, then $\mathbf{R}$ is determined by the lateral ray $\mathbf{R}=\mathbf{R}_{l} k\left(t_{k l}\right)$, since it clearly passes through the lesion.

The above represents the rationale to be used in estimating location and magnification. The major source of error is due to overlapping of projections, which can introduce uncertainties in the value of $\mathbf{R}$ on the order of a lesion dimension. However, such errors are of small consequence in the determination of magnification since only the lesion-aperture distance is important.

The evaluation of $\left[t_{i j}, w_{i j}\right]$ is presented in Appendix A. The results are as follows:

$$
\begin{aligned}
t_{i j}= & -b_{l}+\frac{b_{l}}{D}\left[\left(1-\frac{z_{l}^{i} b_{a}}{a b_{l}}\right)\left(1+\frac{1}{a^{2}}\left|\mathbf{r}_{a}^{j}\right|^{2}\right)\right. \\
& \left.+\left(\frac{b_{a}}{b_{l}}+\frac{y_{a}^{j}}{a}\right)\left(\frac{x_{l}^{i} x_{a}^{j}}{a^{2}}-\left(y_{a}^{j}-z_{l}^{i}\right) / a\right)\right], \\
w_{i j}= & b_{a}-\frac{b_{a}}{D}\left[\left(1-\frac{y_{a}^{j} b_{l}}{a b_{a}}\right)\left(1+\frac{1}{a^{2}}\left|\mathbf{r}_{l}^{i}\right|^{2}\right)\right. \\
& \left.+\left(\frac{b_{l}}{b_{a}}-\frac{z_{l}^{i}}{a}\right)\left(\frac{x_{l}^{i} x_{a}^{j}}{a^{2}}-\left(y_{a}^{j}-z_{l}^{i}\right) / a\right)\right],
\end{aligned}
$$

where the factor $D$ is defined by

$$
\begin{gathered}
D \equiv\left(1+\frac{1}{a^{2}}\left|\mathbf{r}_{l}^{i}\right|^{2}\right)\left(1+\frac{1}{a^{2}}\left|\mathbf{r}_{a}^{j}\right|^{2}\right) \\
-\left(\frac{x_{l}^{i} x_{a}^{j}}{a^{2}}-\left(y_{a}^{j}-z_{l}^{i}\right) / a\right)^{2} .
\end{gathered}
$$

Given a particular vector $\mathbf{R}$ locating one lesion, the lateral and anterior magnifications $M_{l}$ and $M_{a}$, respectively, are given by

$$
\begin{aligned}
& M_{l}=a /\left(b_{l}+y\right), \\
& M_{a}=a /\left(b_{a}-z\right),
\end{aligned}
$$

where $\mathbf{R}=(x, y, z)$, and $M$ denotes the magnification of the image on the detector plane.

In order to test the accuracy of the assumptions and derivation above, a small spherical lesion containing ${ }^{131}$ I was inserted into the neck of a water-filled anatomical head and thorax phantom (ELRAD). ${ }^{3}$ Anterior and lateral views were 
recorded and measurements made as indicated above. Parameters were then input into a lesion-location computer program based upon the above derivation. Results from the program placed the lesion so that the distance of closest approach between the two views was $0.3 \mathrm{~mm}$.

\section{B. Lesion volume}

Next, the estimate of the lesion volume will be considered. This estimate is made by using both views unless there is overlap of one or more lesions in a given view, or the magnification characteristics are poor in one view. In the latter case, only one view is referenced, and the average of results from two possible orientations of an ellipse of revolution are employed. If there are two views, then nothing needs to be assumed about orientation, and a systematically obtained upper bound is found for the lesion volume.

For either case, one first finds the values for the major and minor axes of the projected lesion, whose projection is assumed to be elliptical. A program finds the maximum chord length contained in the ellipse from the determination of the edge. This length is taken as the major axis of the ellipse $2 c$. The program then references the edge detection result again and calculates the ellipse area $A$ in $\mathrm{mm}^{2}$. Finally, the minor axis $2 d$ is found from the equation $A=\pi c d$.

\section{Ellipsoid volume from two orthogonal projections.}

An upper limit on the lesion volume when two views are present is now derived. Hormander ${ }^{8}$ has derived such an expression in the case of two orthogonal projections of near- ly parallel rays. The present analysis is confined to two orthogonal pinhole projections in which some additional complications enter (e.g., magnification). For simplicity, ideal pinhole geometry is once more assumed. The variation of the magnification within a given "lesion" is also neglected. Consider ray vectors $\mathbf{R}_{l}(t)$ and $\mathbf{R}_{a}(w)$, corresponding to the rays passing from the boundary point $\mathbf{R}=(x, y, z)$ of the ellipsoid to the complementary image points $\mathbf{r}_{l}=\left(x_{l}, z_{l}\right)$ and $\mathbf{r}_{a}=\left(x_{a}, y_{a}\right)$. The former are mathematically represented as

$$
\begin{aligned}
& \mathbf{R}_{l}(t)=\left[x\left(\frac{t+b_{l}}{y+b_{l}}\right), t, z\left(\frac{t+b_{l}}{y+b_{l}}\right)\right], \\
& \mathbf{R}_{a}(w)=\left[x\left(\frac{w-b_{a}}{z-b_{a}}\right), y\left(\frac{w-b_{a}}{z-b_{a}}\right), w\right] .
\end{aligned}
$$

In this representation, it is clear that $\mathbf{R}_{l}(y)=\mathbf{R}_{a}(z)=\mathbf{R}$.

The determination of ellipsoid volume from two views requires a knowledge of the ellipse orientation and semiaxes in each projection. Suppose that the ellipse on the lateral plane has semiaxes $c_{l}$ and $d_{l}, c_{l}$ being the larger, and let $\phi_{l}$ denote the angle of rotation of the major axis relative to a line passing through the ellipse center and parallel to the $x$ axis. This arrangement is shown in Fig 3. The corresponding parameters in the anterior view are defined as $c_{a}, d_{a}$, and $\phi_{a}$, respectively. The detailed analysis for volume estimation is presented in Appendix B. The "best" estimate for the lesion volume in terms of the experimental parameters is given by Eq. (B22) as

$$
V \leqslant \frac{4 \pi}{3}\left(M_{a} M_{l}\right)^{-2} c_{l} d_{l} c_{a} d_{a} \min \left[M_{a}\left(c_{a}^{2} \cos ^{2} \phi_{a}+d_{a}^{2} \sin ^{2} \phi_{a}\right)^{-1 / 2}, M_{l}\left(c_{l}^{2} \cos ^{2} \phi_{l}+d_{l}^{2} \sin ^{2} \phi_{l}\right)^{-1 / 2}\right],
$$

where $M_{l}$ and $M_{a}$ are the magnifications specified in Eq. (8), and $\min$ [ ] refers to the smallest element of the set [ ].

\section{Ellipsoid volume from one projection.}

In the case of only one view, a systematic volume estimate, such as given in Eq. (10), does not exist. One is, therefore, limited to treating the lesion as an ellipsoid of rotation. Sup-

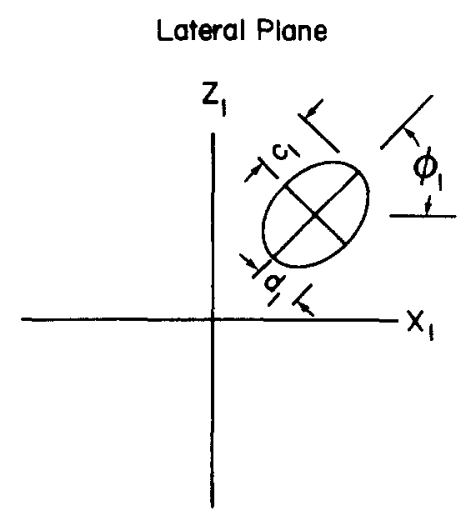

Fig. 3. Idealization of a lesion projection onto the lateral plane as an ellipse characterized by minor and major axes $2 d_{l}$ and $2 c_{l}$, respectively. $\phi_{l}$ is the orientation of the major axis relative to the abscissa. pose that only the lateral projection is available, then the lesion volume is approximated as the mean of the two possible elllipsoids

$$
V=\frac{4 \pi}{6}\left(c_{l}^{2} d_{l}+d_{l}^{2} c_{l}\right)
$$

The replacement $l \rightarrow a$ is made in Eq. (11) if the anterior projection is used.

\section{Uptake}

Since the pinhole collimator is used for imaging, there is an efficiency falloff with angle in the images that must be corrected. Since the falloff depends only on angle, a single image of a mock-iodine ${ }^{133} \mathrm{Ba}$ uniform disk source that is close enough to the pinhole to flood the crystal is sufficient. From this image, one forms an "inverse image" which will, when multiplied element by element, bring the flood to a uniform value. This inverse image is then applied to the patient data.

The efficiency of the camera itself needs to be measured as well. This measurement is made by taking a small source and placing it directly in front of the collimator pinhole and varying the distance from the pinhole while recording an image at each distance. The same edge detection program that is used on the patient data then finds the edge of the object and 
then the counts contained within it. The source activity is measured to within $10 \%$ by a well-type ionization chamber "dose" calibrator. The resulting in-air calibration curve is shown in Fig. 4.

Thereafter, uptake over successive days for each lesion are measured from the pinhole images. If different size regions of interest are found by the edge detection algorithm for the same lesion over different days, then the counts within the region are scaled to a fixed region size. Smoothly varying "background" is significant only on the earliest days and is estimated from a nearby region and subtracted.

The use of the efficiency curve by itself would neglect the effects of the surrounding tissue. By measuring the distance from the pinhole to a point on the surface of the neck, one can calculate the attenuation for water-equivalent tissue for a perpendicular distance from the lesion to a plane tangent to the neck at this point, because the three-dimensional location of the lesion and the pinhole have already been calculated. The curvature of the neck which reduces attenuation and the angle of the radiation path which increases it are compensating errors which are neglected in this model. The apparent uptake determined from the curve is multiplied by the inverse of $e^{-u x}$ with $x$ as the distance discussed above and $u=0.110 \mathrm{~cm}^{-1}$.

In addition, there is the possibility that inscattering of gamma rays from the surrounding tissue into the same path as true events will cause the uptake determined as above to be too high. To check this effect, measurements were made on a plastic head and shoulders phantom at a distance of 11.6 $\mathrm{cm}$ from pinhole to neck surface. A small lesion on a rod was placed at different distances from the neck surface along the pinhole axis, and images recorded at each position with and without water in the phantom. The uptake counts with water present were corrected for $e^{-u x}$ factors which ranged from $0.39-0.84$. The results with corrected counts (water) divided by counts (air) versus depth are shown in Fig. 5 . It is seen that inscattering accounts for an increase of from $7 \%$ to $10 \%$ of

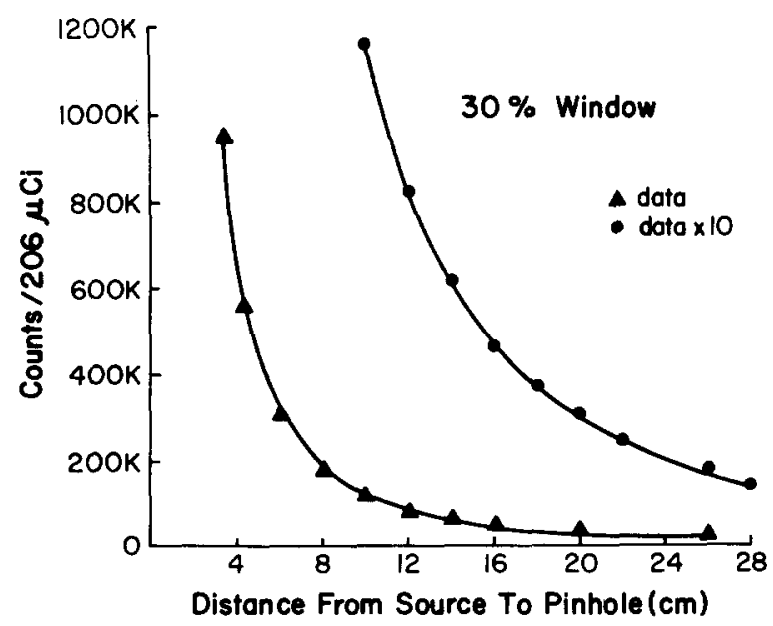

FIG. 4. In-air calibration curve plotting camera counts versus distance of $206-\mu \mathrm{Ci}$ source from pinhole. The dependence is very nearly (distance) ${ }^{-2}$. Camera pulse height window is symmetrically set at $30 \%$. For convenience in reading, the count value times ten are also plotted for the larger distances.

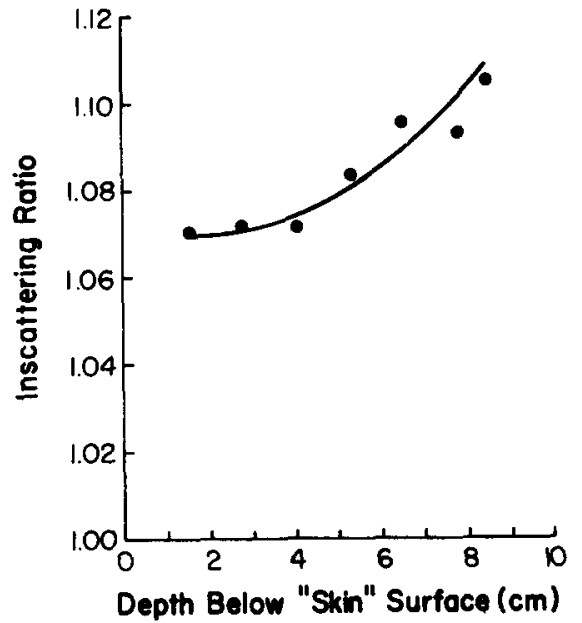

FIG. 5. Inscattering counts are counts which appear within the lesion region due to gamma-ray scattering of surrounding tissue. The ratio of true counts plus inscattering counts over true counts is plotted versus distance below the skin surface. It rises slowly as the lesion is located deeper in the neck. Measurement was made with a plastic head, neck, and shoulders phantom.

the true value. Nevertheless, since a correction for this effect would have required measurements over a wide range of situations and since the error was within the range of similar errors in the uptake, no inscattering correction is applied to the uptake measurement.

\section{Dosimetry}

The uptake measurements are repeated over several days. The measured volume is converted to a mass by assuming a density of $1 \mathrm{gm}$ per cubic centimeter. The computation of total dose is carried out in two time segments.

The last several uptake points are fit with single exponentials to get an effective half-life $T_{\text {eff }}$. The absorbed dose $\bar{D}_{n}(\infty)$, from day $n$, the beginning of the exponential decay, to infinity, is calculated using the following equation:

$$
\bar{D}_{n}(\infty)=\widetilde{C} \sum_{i} \Delta_{i} \phi_{i},
$$

where $\widetilde{C}$, in $\mu \mathrm{Ci} \mathrm{h} / \mathrm{g}$, is the cumulated, concentration in the lesion $\Delta_{i}$, in $\mathrm{g} \mathrm{rad} / \mu \mathrm{Ci} \mathrm{h}$, is the equilibrium absorbed dose constant for radiations of type $i$ and $\phi_{i}$ is the absorbed fraction for radiations of type $i$. For our development, $\widetilde{C}$ is calculated as follows:

$$
\widetilde{C}=34.56 \mathrm{~h} \mathrm{day}^{-1} C_{n} T_{\text {eff }} \text {, }
$$

where $C_{n}$, in $\mu \mathrm{Ci} / \mathrm{g}$, is the uptake concentration in the lesion at day $n$ and $T_{\text {eff }}$ is the effective half-life, in days, for the lesion. For ${ }^{131} \mathrm{I}$, the sum of the $\Delta_{i} \phi_{i} s$ for nonpenetrating radiation is 0.4135 . For penetrating radiations, assuming a 0.03 approximation for $\phi_{i}$, the sum is 0.024 . The equation thus becomes

$$
\bar{D}_{n}(\infty)=15.12 C_{n} T_{\text {eff }} \text {. }
$$

The first several uptake values are converted to dose rates in $\mathrm{rad} / \mathrm{h}$ in a manner consistent with the assumptions above. They are integrated graphically to produce $\bar{D}_{0}(n)$.

The final dose in rad is then the sum of $\bar{D}_{0}(n)$ plus $\bar{D}_{n}(\infty)$. 
TABLE I. Distance of closest approach when associating projected lesions from two orthogonal views.

\begin{tabular}{lcc}
\hline \hline \multicolumn{2}{c}{ Projected lesion number } \\
$\begin{array}{l}\text { Lateral } \\
\text { Anterior } \\
\text { view }\end{array}$ & view & Distance of closest approach \\
\hline 1 & 1 & \\
2 & 1 & $1.4 \mathrm{~mm}^{\mathrm{a}}$ \\
3 & 1 & 20.9 \\
1 & 2 & 25.4 \\
2 & 2 & $7.0^{\mathrm{a}}$ \\
3 & 2 & $4.3^{\mathrm{a}}$ \\
\hline
\end{tabular}

aMinimum values which associate projected lesion numbers in two views as belonging to same lesion.

\section{SAMPLE RESULT}

A sample result for a given patient is now presented in which $175 \mu \mathrm{Ci}$ of ${ }^{131} \mathrm{I}$ was administered. Three lesion projections were clearly separated in the anterior image and were numbered. (1), (2), and (3) from top to bottom. In the lateral view, only two lesion projections were visible and were numbered (1) and (2). The results of the lesion-location program in this case are shown in Table I. It is seen that anteriorprojected lesion (1) is clearly associated with lateral-projected lesion number (1), since the distance of closest approach is more than 18 times smaller than the distance when it is associated with lateral projected lesion number (2) $(1.4 \mathrm{~mm}$ compared to $25.4 \mathrm{~mm}$ ). Anterior-projected lesion number (2) is less surely associated with lateral-projected lesion number (2) $(7.0 \mathrm{~mm}$ compared to $12.8 \mathrm{~mm}$ ) and anterior-projected lesion number $(3)$ is associated with lateral projected lesion number (2) with intermediate confidence $(4.3 \mathrm{~mm}$ versus $20.9 \mathrm{~mm}$ ). Part of the error in locating anterior-projected lesion numbers (2) or (3) is due to the uncertainty of choosing a location value for the overlap of two lesions.

From here on, the anterior-projected lesion number will be called the lesion number. For this patient, lesion (1) has an upper limit calculated for its volume, while lesions (2) and (3) are only specified to an approximate volume. Input parameters for Eqs. (10) and (11) are given in Table II. In addition, for lesion (1), $\phi_{a}=56^{\circ}$ and $\phi_{l}=20.5^{\circ}$. The computed volumes are given in the last column of the table.

For each lesion, the time behavior of the absolute uptake is found in Fig. 6 as well as the integrated dose up to day 4 and the integrated dose from day 4 to infinity. Total dose values are found in the last column of Table III.

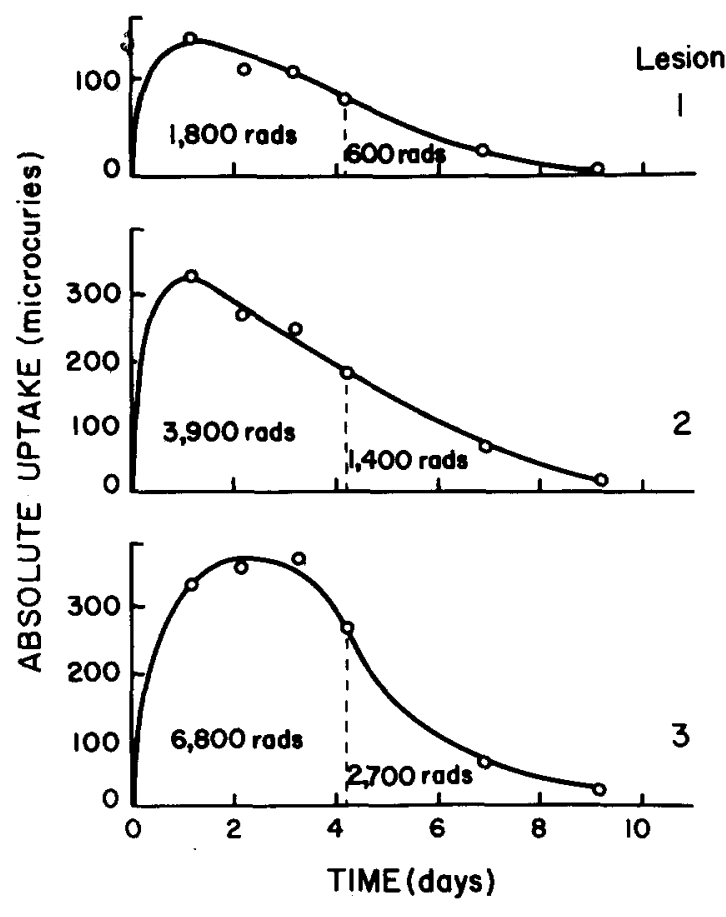

FIG. 6. Sample result of a patient with three lesions who was administered 175 millicuries of ${ }^{131} \mathrm{I}$. Uptake in microcuries for each lesion is plotted versus time after the administration of the iodine. A single exponential decay was fitted to the last three data points for each lesion. The dose in rad to the lesions over the first four days and that over four days to infinity are shown under the pertinent parts of each curve.

\section{DISCUSSION}

A dose calculation method that involves anterior and lateral views with a pinhole collimator mounted on an Anger camera and an edge detection algorithm is given. A method for lesion location and volume estimation is described. Values of rad dose are calculated lesion by lesion. The largest source of error in the absolute accuracy of the rad dose calculation is felt to be error in the determination of the volume. Further investigation of the behavior of the edge detection algorithm and the resulting volume estimate as a function of magnification and size would be helpful in reducing lesion to lesion variation. Evaluation of the usefulness of the current model awaits correlation of measured rad dose to clinical condition one year post therapy in a sample of patients.

The time dependence displayed in Fig. 6 is in general agreement with Wollman's model for the accumulation of

TABLE II. Lesion sizing.

\begin{tabular}{|c|c|c|c|c|c|c|c|}
\hline $\begin{array}{l}\text { Lesion } \\
\text { number }\end{array}$ & $\begin{array}{l}\text { Magnification } \\
M_{a}\end{array}$ & $\begin{array}{l}\text { terior } \\
\text { Semimajor axis } \\
\text { of projected } \\
\text { lesion } \\
c_{a}\end{array}$ & $\begin{array}{l}\text { Semiminor axis } \\
\text { of projected } \\
\text { lesion } \\
d_{a}\end{array}$ & $\begin{array}{l}\text { Magnification } \\
M_{\ell}\end{array}$ & $\begin{array}{l}\text { Semimajor axis } \\
\text { of projected } \\
\text { lesion } \\
c_{\text {l }}\end{array}$ & $\begin{array}{l}\text { Semiminor axis } \\
\text { of projected } \\
\text { lesion } \\
d_{i}\end{array}$ & Volume \\
\hline 1 & 3.1 & $2.28 \mathrm{~cm}$ & $1.81 \mathrm{~cm}$ & 1.0 & $1.79 \mathrm{~cm}$ & $1.23 \mathrm{~cm}$ & $2.9 \mathrm{~cm}^{3 \mathrm{a}}$ \\
\hline 2 & 2.1 & 2.11 & 1.65 & 0.84 & $\ldots$ & ... & $2.9^{\mathrm{b}}$ \\
\hline 3 & 2.1 & 1.90 & 1.48 & 0.93 & $\ldots$ & $\cdots$ & $2.11^{\mathrm{b}}$ \\
\hline
\end{tabular}

This value represents an upper bound.

${ }^{b}$ These values represent best approximate results. 
TABLE III. Dose calculation.

\begin{tabular}{lllll}
\hline \hline $\begin{array}{l}\text { Lesion } \\
\text { number }\end{array}$ & $\begin{array}{l}\text { Pinhole to lesion } \\
\text { perpendicular distance }\end{array}$ & $\begin{array}{l}\text { Skin surface to lesion } \\
\text { perpendicular distance }\end{array}$ & $\begin{array}{l}\text { Effective half- } \\
\text { life } T_{\text {eff }}\end{array}$ & Dose \\
\hline 1 & $6.12 \mathrm{~cm}$ & $1.44 \mathrm{~cm}$ & 1.42 days & $2400 \mathrm{rad}^{\mathrm{a}}$ \\
2 & 9.02 & 4.34 & 1.52 & $5300^{\mathrm{b}}$ \\
3 & 8.96 & 4.28 & 1.43 & $9500^{\mathrm{b}}$ \\
\hline
\end{tabular}

${ }^{a}$ This value represents a lower limit.

'These values represent best approximate results.

iodine in metastatic lesions wherein the peak in time can occur at 1-20 days, depending on the parameters used. ${ }^{9}$ For a bone lesion and a therapy activity of ${ }^{131} \mathrm{I}$, Marcuse $e t$ al. show a smooth interpolation of data at $5 \mathrm{~h}, 2$ and 4 days that has a peak near 2 days. ${ }^{9}$ They use a single exponential fit of their data from day 2 on and point out that the inclusion of an earlier measurement decreases the dose from that which would be calculated by extrapolating the single exponential to time zero. For tracer activities of administered ${ }^{131} I$ in thyroid lesions, on the other hand, Thomas et al. ${ }^{3}$ show a typical case that can be fit with a single exponential from day 1 through day 3 and they extrapolate this exponential back to time zero. The early-uptake behavior of ${ }^{131} \mathrm{I}$, therefore, is not yet established.

\section{ACKNOWLEDGMENTS}

This work was supported by NIH grant Nos. 1 RO1 GM28305-01 and 5T32-CA09015-05. The authors thank Diane Vecellio for secretarial assistance in preparation of the manuscript and William Downer for technical assistance in fabrication of the head, neck, and shoulders phantom.

\section{APPENDIX A: EVALUATION OF $\left(t_{i j}, w_{i j}\right)$}

In terms of Eqs. (3) and (4), the square displacement $S_{i j}^{2}(t, w)$ is given by

$$
\begin{aligned}
& S_{i j}^{2}(t, w) \\
& =\left[x_{l}^{i}\left(\frac{t+b_{l}}{a}\right)+x_{a}^{j}\left(\frac{w-b_{a}}{a}\right)\right]^{2}+\left[t-y_{a}^{j}\left(\frac{w-b_{a}}{a}\right)\right]^{2} \\
& \quad+\left(z_{l}^{i}\left(\frac{t+b_{l}}{a}\right)+w\right)^{2} .
\end{aligned}
$$

The minimum of Eq. (A1) is found by first observing the two linear equations, obtained by setting

$$
\begin{aligned}
& \frac{\partial}{\partial t} S_{i j}^{2}(t, w)=0 \\
& \frac{\partial}{\partial w} S_{i j}^{2}(t, w)=0 .
\end{aligned}
$$

The resulting equations are

$$
\begin{aligned}
& \left(t+b_{l}\right)\left(1+\frac{1}{a^{2}}\left|\mathbf{r}_{l}^{i}\right|^{2}\right)+\left(w-b_{a}\right)\left[\frac{x_{a}^{j} x_{l}^{i}}{a^{2}}-\left(y_{a}^{j}-z_{l}^{i}\right) / a\right] \\
& \quad=\left(b_{l}-b_{a} z_{l}^{i} / a\right), \\
& \left(t+b_{l}\right)\left[\frac{x_{a}^{j} x_{l}^{i}}{a^{2}}-\left(y_{a}^{j}-z_{l}^{i}\right) / a\right]+\left(w-b_{a}\right)\left(1+\frac{1}{a^{2}}\left|\mathbf{r}_{a}^{j}\right|^{2}\right) \\
& =-\left(b_{a}+b_{l} y_{a}^{j} / a\right) .
\end{aligned}
$$

The equations are readily solved, yielding the following expression for $\left(t_{i j}, w_{i j}\right)$ :

$$
\begin{aligned}
& t_{i j}=-b_{l}+\frac{b_{l}}{D}\left[\left(1-\frac{z_{l}^{i} b_{a}}{a b_{l}}\right)\left(1+\frac{1}{a^{2}}\left|\mathbf{r}_{a}^{j}\right|^{2}\right)+\left(\frac{b_{a}}{b_{l}}+\frac{y_{a}^{j}}{a}\right)\left(\frac{x_{l}^{i} x_{a}^{j}}{a^{2}}-\left(y_{a}^{j}-z_{l}^{i}\right) / a\right)\right], \\
& w_{i j}=b_{a}-\frac{b_{a}}{D}\left\{\left(1+\frac{y_{a}^{j} b_{l}}{a b_{a}}\right)\left(1+\frac{1}{a^{2}}\left|\mathbf{r}_{l}^{i}\right|^{2}\right)+\left(\frac{b_{l}}{b_{a}}-\frac{z_{l}^{i}}{a}\right)\left[\frac{x_{l}^{i} x_{a}^{j}}{a^{2}}-\left(y_{a}^{j}-z_{l}^{i}\right) / a\right]\right\}
\end{aligned}
$$

where the factor $D$ is defined by

$$
\begin{aligned}
D \equiv & \left(1+\frac{1}{a^{2}}\left|\mathbf{r}_{l}^{i}\right|^{2}\right)\left(1+\frac{1}{a^{2}}\left|\mathbf{r}_{a}^{j}\right|^{2}\right) \\
& -\left[\frac{x_{a}^{j} x_{l}^{i}}{a^{2}}-\left(y_{a}^{j}-z_{l}^{i}\right) / a\right]^{2} .
\end{aligned}
$$

\section{APPENDIX B: VOLUME ESTIMATION FROM TWO VIEWS}

The equation of an ellipsoid centered at the point $\mathbf{R}=\mathbf{B}=\operatorname{col}\left(B_{x}, B_{y}, B_{z}\right)$ is given in matrix notation by

$$
(\mathbf{R}-\mathbf{B})^{T} \cdot \mathbf{A} \cdot(\mathbf{R}-\mathbf{B})=1 \text {, }
$$

where col refers to column, and $\mathrm{A}$ is symmetric with positive eigenvalues and elements $\left[A_{i j}\right], i, j=1,2,3$. Consider first the projection of Eq. (B1) onto the lateral plane. The anterior projection then follows by inspection.

The lateral projection of the ellipsoid [Eq. (B1)] is obtained by using Eq. (9a) with $t=-\left(b_{l}+a\right)$ and noting the relation

$$
\left(\begin{array}{l}
x \\
z
\end{array}\right)=-\left(\frac{y+b_{l}}{a}\right) \mathbf{r}_{l} \text {. }
$$

The following definitions are introduced:

$$
\begin{aligned}
& \mathbf{A}_{l}=\left(\begin{array}{ll}
A_{11} & A_{13} \\
A_{31} & A_{33}
\end{array}\right), \\
& \mathbf{B}_{l}^{(0)}=\frac{a}{y+b_{l}}\left(\begin{array}{l}
B_{x} \\
B_{z}
\end{array}\right),
\end{aligned}
$$




$$
\begin{aligned}
& \mathbf{B}_{l}^{(1)}=\frac{a}{y+b_{l}}\left(\begin{array}{l}
A_{12} \\
A_{32}
\end{array}\right), \\
& \mathbf{B}_{l}=\mathbf{B}_{l}^{(0)}-\left(y-B_{y}\right) \mathbf{A}_{l}^{-1} \cdot \mathbf{B}_{l}^{(1)} .
\end{aligned}
$$

Equations $(\mathrm{B} 1)-(\mathrm{B} 3)$ are then combined, resulting in

$$
\begin{aligned}
\left(\mathbf{r}_{l}+\mathbf{B}_{l}\right)^{T} \cdot \mathrm{A}_{l} \cdot\left(\mathbf{r}_{l}+\mathbf{B}_{l}\right)= & \left(\frac{a}{y+b_{l}}\right)^{2}\left[1-A_{22}\left(y-B_{y}\right)^{2}\right] \\
& +\left(y-B_{y}\right)^{2} \mathbf{B}_{l}^{(1)^{T}} \mathbf{A}_{l}^{-1} \cdot \mathbf{B}_{l}^{(1)} .
\end{aligned}
$$

From Fq. (B4), it is evident that each slice of constant $y$ in the object distribution projects onto an ellipse in the lateral plane centered at $\mathbf{r}_{l}=-\mathbf{B}_{l}$.

Neglect of the variation in magnification within the ellipsoid amounts to replacing $a /\left(y+b_{l}\right)$ by $a /\left(B_{y}+b_{l}\right)$. Thus, for example, $\mathbf{B}_{l}^{(0)}$ is set equal to $\left[a /\left(B_{y}+b_{l}\right)\right] \operatorname{col}[B x, B x]$.

Similar results can be written for the anterior plane as follows: noting that Eq. (B2) must be replaced by

$$
\left(\begin{array}{l}
x \\
y
\end{array}\right)=-\left(\frac{b_{a}-B_{z}}{a}\right) \mathbf{r}_{a},
$$

results in

$$
\begin{aligned}
\left(\mathbf{r}_{a}\right. & \left.+\mathbf{B}_{a}\right)^{T} \cdot \mathbf{A}_{a} \cdot\left(\mathbf{r}_{a}+\mathbf{B}_{a}\right)=\left(\frac{a}{b_{a}-B_{z}}\right)^{2}\left[1-A_{33}\left(z-B_{z}\right)^{2}\right] \\
& +\left(z-B_{z}\right)^{2} \mathbf{B}_{a}^{(1)} \cdot \mathbf{A}_{a}^{-1} \cdot \mathbf{B}_{a}^{(1)},
\end{aligned}
$$

where

$$
\begin{aligned}
& \mathrm{A}_{a}=\left(\begin{array}{ll}
A_{11} & A_{12} \\
A_{21} & A_{22}
\end{array}\right), \\
& \mathbf{B}_{a}^{(0)}=\left(\frac{a}{b_{a}-B_{z}}\right)\left(\begin{array}{l}
B_{x} \\
B_{y}
\end{array}\right), \\
& \mathbf{B}_{a}^{(1)}=\left(\frac{a}{b_{a}-B_{z}}\right)\left(\begin{array}{l}
A_{13} \\
A_{23}
\end{array}\right), \\
& \mathbf{B}_{a}=\mathbf{B}_{a}^{(0)}-\left(z-B_{z}\right) A_{a}^{-1} \cdot \mathbf{B}_{a}^{(1)} .
\end{aligned}
$$

The magnification approximation is already introduced into Eqs. (B5)-(B7).

The image appearing on the lateral plane results from the superposition of many projected slices (an infinite number) through the object distribution. The boundary of the lateral image then consists of the locus of extrema points of this superposition. Regarding one such slice image as $z_{l}=z_{l}\left(x_{l}, y\right)$, a boundary point is obtained by finding the value of $y$, for which

$$
\frac{\partial}{\partial y} z_{l}\left(x_{l}, y\right)=0
$$

The value of $y$ for which Eq. (B8) is satisfied is obtained directly from differentiation of Eq. (B4). The result is

$$
y-B_{y}=\left(\frac{B_{y}+b_{l}}{a}\right)^{2} \frac{1}{A_{22}} \mathbf{B}_{l}^{(1)^{T}} \cdot\left[\mathbf{r}_{l}+\mathbf{B}_{l}^{(0)}\right] .
$$

Substitution of Eq. (B9) in Eq. (B4) yields an equation for the experimentally observed ellipse. Defining a new matrix $A_{l}^{\text {exp }}$, we find

$$
\left[\mathbf{r}_{l}+\mathbf{B}_{l}^{(0)}\right]^{T} \cdot A_{l}^{\exp } \cdot\left[\mathbf{r}_{l}+\mathbf{B}_{l}^{(0)}\right]=1,
$$

$$
\mathrm{A}_{l}^{\exp } \equiv\left(\frac{B_{y}+b_{l}}{a}\right)^{2}\left[\mathrm{~A}_{l}-\frac{1}{A_{22}}\left(\frac{B_{y}+b_{l}}{a}\right)^{2} \mathbf{B}_{l}^{(1)} \mathbf{B}_{l}^{(1)^{T}}\right]
$$

Thus, the observed ellipse is related to the ellipsoid through the quantities $A_{l}^{\exp }$ and $B_{l}^{(0)}$. The observed semiaxes are, in turn, directly related to the components of $A_{l}^{\exp }$.

Equations analogous to Eqs. (B10) and (B11) in the anterior view are written by inspection

$$
\begin{aligned}
& {\left[\mathbf{r}_{a}+\mathbf{B}_{a}^{(0)}\right]^{T} \cdot \mathrm{A}_{a}^{\exp } \cdot\left[\mathbf{r}_{a}+\mathbf{B}_{a}^{(0)}\right]=1,} \\
& \mathrm{~A}_{a}^{\exp } \equiv\left(\frac{B_{z}-b_{a}}{a}\right)^{2}\left[\mathrm{~A}_{a}-\frac{1}{A_{33}}\left(\frac{B_{z}-b_{a}}{a}\right)^{2} \mathbf{B}_{a}^{(1)} \mathbf{B}_{a}^{(1)^{T}}\right],
\end{aligned}
$$

where $A_{a}, B_{a}^{(0)}$, and $B_{a}^{(1)}$ are given by Eq. (B7). Note also that the magnification appropriate to the anterior plane is used.

A relationship is now derived relating the components of $A$ to the observed ellipse semiaxes and orientation. Without loss of generality, we can set $\mathbf{B}_{l}^{(0)}=0$ and consider the rotation matrix which places Eq. (B10) into canonical form. Suppose that the ellipse has semiaxes $c_{l}$ and $d_{l}, c_{l}$ being the larger, and $\phi_{l}$ is the angle of rotation to the major axis relative to the abscissa. The canonical form of $A_{l}^{\text {exp }}$ and Eq. (B11) are then related by the expression

$$
\left(\begin{array}{cc}
1 / c_{l}^{2} & 0 \\
0 & 1 / d_{l}^{2}
\end{array}\right)=\mathrm{R}_{l} \cdot \mathrm{A}_{l}^{\exp } \cdot \mathrm{R}_{l}^{T}
$$

where

$$
\mathrm{R}_{l}=\left(\begin{array}{cc}
\cos \phi_{l} & \sin \phi_{l} \\
-\sin \phi_{l} & \cos \phi_{l}
\end{array}\right) \text {. }
$$

Using the orthogonality property of the rotation matrix, $\mathrm{R}_{l} \cdot \mathrm{R}_{l}^{T}=\mathrm{I}$, I being the unit matrix, the components of $\mathrm{A}_{l}^{\text {exp }}$ are readily expressed in terms of measureable quantities

$$
\begin{aligned}
A_{l 11}^{\exp } & =\left(\frac{b_{l}+B_{y}}{a}\right)^{2}\left(A_{11}-\frac{A_{12}^{2}}{A_{22}}\right) \\
& =c_{l}^{-2} \cos ^{2} \phi_{l}+d_{l}^{-2} \sin ^{2} \phi_{l}, \\
A_{l 33}^{\exp } & =\left(\frac{b_{l}+B_{y}}{a}\right)^{2}\left(A_{33}-\frac{A_{32}^{2}}{A_{22}}\right) \\
& =c_{l}^{-2} \sin ^{2} \phi_{l}+d_{l}^{-2} \cos ^{2} \phi_{l}, \\
A_{l 13}^{\exp } & =\left(\frac{b_{l}+B_{y}}{a}\right)^{2}\left(A_{13}-\frac{A_{12} A_{23}}{A_{22}}\right) \\
& =\frac{1}{2}\left(c_{l}^{-2}-d_{l}^{-2}\right) \sin 2 \phi_{l} .
\end{aligned}
$$

The corresponding relations for the anterior plane are given by

$$
\begin{aligned}
A_{a 11}^{\exp } & =\left(\frac{b_{a}-B_{z}}{a}\right)^{2}\left(A_{11}-\frac{A_{13}^{2}}{A_{33}}\right) \\
& =c_{a}^{-2} \cos ^{2} \phi_{a}+d_{a}^{-2} \sin ^{2} \phi_{a} \\
A_{a_{22}}^{\exp } & =\left(\frac{b_{a}-B_{z}}{a}\right)^{2}\left(A_{22}-\frac{A_{23}^{2}}{A_{33}}\right) \\
& =c_{a}^{-2} \sin ^{2} \phi_{a}+d_{a}^{-2} \cos ^{2} \phi_{a}, \\
A_{a 12}^{\exp } & =\left(\frac{b_{a}-B_{z}}{a}\right)^{2}\left(A_{12}-\frac{A_{13} A_{32}}{A_{33}}\right)
\end{aligned}
$$




$$
=\frac{1}{2}\left(c_{a}^{-2}-d_{a}^{-2}\right) \sin 2 \phi_{a} .
$$

Only 5 of the 6 equations in Eqs. (B16) and (B17) are independent and, therefore, the ellipsoid is not uniquely specified with two pinhole projections. However, a volume estimate is still possible. Consider, for example, the area of lateral projection

$$
\pi c_{l} d_{l}=\pi\left(\operatorname{det} \mathrm{A}_{l}^{\mathrm{exp}}\right)^{-1 / 2}
$$

where the prefix det refers to the determinant. Equation (B18) is derived directly from Eq. (B14), using the multiplicity properties of determinants and orthogonality of $R_{l}$. But from Eq. (B16), we find $\operatorname{det} \mathrm{A}_{l}^{\exp }=\left(\frac{b_{l}+B_{y}}{a}\right)^{2} \frac{1}{A_{22}} \operatorname{det} \mathrm{A}$.

The volume $V$ of the ellipsoid is, on the other hand, given by the expression

$$
V=\frac{4 \pi}{3}(\operatorname{det} A)^{-1 / 2}
$$

which upon substitution of Eq. (B19) reduces to

$$
V=\frac{4 \pi}{3}\left(\frac{b_{l}+B_{y}}{a}\right)^{2} A_{22}^{-1 / 2}\left(\operatorname{det} A_{l}^{\exp }\right)^{-1 / 2}
$$

Equation (B20) indicates that knowledge of $A_{22}$ is required to give the lesion volume. The element $A_{22}$ is directly estimated from the information on the anterior projection, Eq. (B17),

$$
\begin{aligned}
V= & \frac{4 \pi}{3}\left(\frac{b_{l}+B_{y}}{a}\right)^{2} c_{l} d_{l}\left[\left(\frac{a}{b_{a}-B_{z}}\right)^{2}\left(c_{a}^{-2} \sin ^{2} \phi_{a}+d_{a}^{-2} \cos ^{2} \phi_{a}\right)+A_{23}^{2} / A_{33}\right]^{-1 / 2} \\
& \leqslant \frac{4 \pi}{3}\left(\frac{b_{l}+B_{y}}{a}\right)^{2}\left(\frac{b_{a}-B_{z}}{a}\right) c_{l} d_{l} c_{a} d_{a}\left[d_{a}^{2} \sin ^{2} \phi_{a}+c_{a}^{2} \cos ^{2} \phi_{a}\right]^{-1 / 2}
\end{aligned}
$$

Thus, Eq. (B21) constitutes an upper bound to the lesion volume involving experimental information from both lateral and anterior views. Furthermore, an equation similar to Eq. (B21) would follow starting from the information contained in det $A_{a}^{\text {exp }}$. Since both expressions give upper bounds, the best volume estimate is then the least upper bound, or

$$
\begin{aligned}
& V \leqslant \frac{4 \pi}{3}\left(\frac{b_{l}+B_{y}}{a}\right)^{2}\left(\frac{b_{a}-B_{z}}{a}\right)^{2} c_{l} d_{l} c_{a} d_{a} \text { minimum }\left[\frac{a}{b_{a}-B_{z}}\left(c_{a}^{2} \cos ^{2} \phi_{a}+d_{a}^{2} \sin ^{2} \phi_{a}\right)^{-1 / 2},\right. \\
& \left.\frac{a}{b_{l}+B_{y}}\left(c_{l}^{2} \cos ^{2} \phi_{l}+d_{l}^{2} \sin ^{2} \phi_{l}\right)^{-1 / 2}\right] .
\end{aligned}
$$

${ }^{1}$ J. S. Scott, K. E. Halnan, J. Shimmins, P. Kostaki, and H. McKenzie, Br. J. Radiol. 43, 256 (1970).

${ }^{2}$ S. R. Thomas, H. R. Maxon, and J. G. Kereiakes, Med. Phys. 3, 253 (1975).

${ }^{3}$ S. R. Thomas, H. R. Maxon, J. G. Kereiakes, and E. L. Saenger, Radiology 122, 731 (1977).

${ }^{4}$ L. T. Kircos, "Qualitative Organ Visualization," Ph.D. thesis, University of Michigan (1978).

${ }^{5}$ J. W. Keyes, Jr., N. Orlandea, W. J. Heetderks, P. F. Leonard, and W. L. Rogers, J. Nucl. Med. 18, 381 (1977).

${ }^{6} \mathrm{R}$. Loevinger and M. Berman, $A$ Revised Schema for Calculating the Ab- sorbed Dose from Biologically Distributed Radionuclides, MIRD Pamphlet No. 1, Revised (Society of Nuclear Medicine, New York, 1976).

${ }^{7}$ W. Ellett and R. Humes, Absorbed Fractions for Small Volumes Containing Photon-Emitting Radioactivity, MIRD Suppl. No. 5, Pamphlet No. 8 (Society of Nuclear Medicine, New York, 1971).

${ }^{8}$ Analysis by L. Hormander in review article by H. Arvidsson, Acta Radiol. Suppl. 158, 33 (1958).

${ }^{9}$ H. R. Marcuse, C. C. Delprat, J. F. M. Delemane, C. A. Hoefnagel, H. Muessen, and J. B. Van der Shoot in Proceedings of the Third International Radiopharmaceutical Dosimetry Symposium, Oak Ridge TN (1981), p. 595. 